Pacific

Journal of

Mathematics

THE CHERN-SIMONS INVARIANTS FOR THE DOUBLE OF A COMPRESSION BODY

DAVID L. DUNCAN 


\title{
THE CHERN-SIMONS INVARIANTS FOR THE DOUBLE OF A COMPRESSION BODY
}

\author{
DAVID L. DUNCAN
}

Given a 3-manifold that can be written as the double of a compression body, we compute the Chern-Simons critical values for arbitrary compact connected structure groups. We also show that the moduli space of flat connections is connected when there are no reducibles.

1. Introduction 17

2. Background 20

3. Chern-Simons values and instantons 25

$\begin{array}{ll}\text { Acknowledgments } & 37\end{array}$

$\begin{array}{ll}\text { References } & 37\end{array}$

\section{Introduction}

Let $G$ be a Lie group with Lie algebra $\mathfrak{g}$. Given a principal $G$-bundle $P \rightarrow Y$ over a closed, oriented 3-manifold $Y$, one can define the Chern-Simons function

$$
\mathcal{C S}: \mathcal{A}(P) \rightarrow \mathbb{R} / \mathbb{Z}
$$

where $\mathcal{A}(P)$ is the space of connections on $P$. The set of critical points of $\mathcal{C S}$ is the space of flat connections $\mathcal{A}_{\text {flat }}(P) \subset \mathcal{A}(P)$, and the critical values are topological invariants of $Y$. In general, computing the critical values of $\mathcal{C S}$ is fairly difficult. Nevertheless, various techniques have been developed to handle certain classes of 3manifolds; for example, see [Kirk and Klassen 1993; Auckly 1994; Reznikov 1996; Nishi 1998; Neumann and Yang 1995; Dostoglou and Salamon 1994; Wehrheim 2006]. Most of these techniques are specific to the choice of Lie group $G$, common examples being $\mathrm{SU}(2), \mathrm{Sp}(1)$ and $\mathrm{SL}_{\mathbb{C}}(2)$.

In the present paper we compute the Chern-Simons critical values for any 3manifold $Y$ that can be written as a double

$$
Y=\bar{H} \cup_{\partial H} H,
$$

MSC2010: primary 53C07; secondary 53C05.

Keywords: Chern-Simons invariants. 
where $H$ is a compression body, $\bar{H}$ is a copy of $H$ with the opposite orientation, and the identity map on $\partial H$ is used to glue $\bar{H}$ and $H$; see Figure 1 . For us, the term compression body means that

- $H$ is a compact, connected, oriented cobordism between surfaces $\Sigma_{-}, \Sigma_{+}$,

- $H$ admits a Morse function $f: H \rightarrow[-1,1]$ with critical points of index 0 or 1 ,

- all critical values of $f$ are in the interior of $(-1,1)$, and

- $f^{-1}( \pm 1)=\Sigma_{ \pm}$.

It follows that, up to homotopy, $H$ can be obtained from $\Sigma_{+}$by attaching 2-handles. These topological assumptions imply that $\Sigma_{+}$is connected; there is no bound on the number of components of $\Sigma_{-}$. (Note that not every 3-manifold can be realized as the double of a compression body; the Poincare homology sphere is a simple counterexample.)

Throughout this paper we work with an arbitrary compact, connected Lie group $G$, and we assume the bundle $P$ is obtained by doubling a bundle over $H$ in the obvious way.
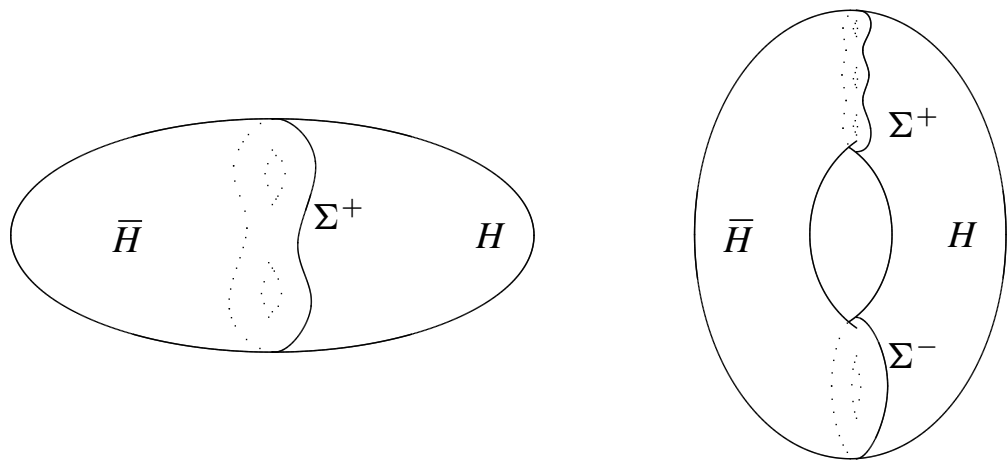

Figure 1. Pictured above are two possibilities for $Y$. The first has $\Sigma_{-}=\varnothing$, making $H$ a handlebody. In the second figure, $\Sigma_{-}$is connected.

Before stating the main result, we mention that the definition of $\mathcal{C S}$ requires a choice of normalization. When $G$ is simple this choice can be made in an essentially unique way. However, for arbitrary compact $G$ the situation is not as simple. It turns out that, in general, this normalization can be fixed by choosing a faithful unitary representation $\rho_{0}: G \rightarrow \mathrm{U}(W)$, where $W$ is a finite-dimensional Hermitian vector space. One upshot of this approach is that certain computations reduce to the case where $G$ is a classical group; see Remark 2.2. It is convenient to phrase the main result in terms of a lift $\mathcal{C S}_{a}: \mathcal{A}(P) \rightarrow \mathbb{R}$ of the Chern-Simons function $\mathcal{C S}$; this lift can be defined by fixing a flat reference connection $a \in \mathcal{A}_{\text {flat }}(P)$. See Section 2B for more details. 
Theorem 1.1. Let $G$ be a compact, connected Lie group. There is a positive integer $N_{G}$ such that if $H, P, Y, \rho_{0}$ are as above, then all critical values of $\mathcal{C} \mathcal{S}_{a}: \mathcal{A}(P) \rightarrow \mathbb{R}$ are integer multiples of $1 / N_{G}$.

The dependence of these critical values on the choice of $\rho_{0}$ is only up to an overall integer multiple. In particular, if the representation $\rho_{0}$ has image in $\mathrm{SU}(W) \subset \mathrm{U}(W)$, then all critical values are multiples of $2 / N_{G}$. If $\rho_{0}$ is the complexification of a faithful orthogonal representation of $G$ (see Remark 2.2), then all critical values are multiples of $4 / N_{G}$.

Following Wehrheim [2006], the integer $N_{G}$ appearing in Theorem 1.1 can be defined explicitly as follows. Consider the integer

$$
n_{G}:=\sup _{G^{\prime} \leq G}\left\{\left|\pi_{0}\left(C\left(G^{\prime}\right)\right)\right|\right\},
$$

where the supremum is over all subgroups of $G$, and $C\left(G^{\prime}\right)$ denotes the centralizer in $G$. Then $n_{G}$ is finite since $G$ is compact. We define $N_{G}$ to be the least common multiple of $\left\{1,2, \ldots, n_{G}\right\}$. Thus $N_{G} \geq 1$ is an integer depending only on $G$.

The definition of $N_{G}$ can often be refined if one has certain knowledge about $G$ or $P$. In particular, the proof will show that we can take $N_{G}=1$ provided the following hypothesis holds.

Hypothesis 1. For each connected component $S \subset \Sigma_{-}$, the identity component of the gauge group acts trivially on $\mathcal{A}_{\text {flat }}\left(\left.P\right|_{S}\right)$.

For example, Hypothesis 1 holds trivially when $\Sigma_{-}$is empty. When $\Sigma_{-}$is nonempty, the hypothesis holds when $G=\mathrm{SO}(3)$ and the restriction of $P$ to each component of $\Sigma_{-}$is nontrivial. More generally, this hypothesis is satisfied if $G=\mathrm{U}(r)$ or PU $(r)$ and the integer $c_{1}(P)[S]$ is coprime to $r$ for all connected components $S \subset \Sigma_{-}$; see [Wehrheim and Woodward 2009]. On the other hand, Hypothesis 1 is never satisfied if the bundle $P$ is trivial, due to the trivial connection. That being said, it is perhaps worth mentioning that there are other hypotheses that allow one to replace $N_{G}$ by 1 . For example, an argument by Wehrheim [2006] can be used in our proof below to show that when $G=\mathrm{SU}(2)$, one can always replace $N_{\mathrm{SU}(2)}$ by 1 in the statement of Theorem 1.1. We also point out that Hypothesis 1 is not assumed in Theorem 1.1; our primary motivation for introducing this hypothesis is to simplify the discussion at various times.

Motivated by the techniques of [Dostoglou and Salamon 1994, page 633] and [Wehrheim 2006], our strategy for proving Theorem 1.1 is to show that all flat connections are gauge equivalent to a connection in a certain canonical form. As a consequence, Theorem 1.1 can be viewed as a statement about the connected components of $\mathcal{A}_{\text {flat }}(P)$. For example, we arrive at the following corollary; see Remark 3.5. 
Corollary 1.2. Let $P \rightarrow Y$ be as in Theorem 1.1. Assume Hypothesis 1 is satisfied and either

- $G=\mathrm{U}(r)$ or $\mathrm{SU}(r)$ and $\rho_{0}$ is the standard representation, or

- $G=\mathrm{PU}(r)$ and $\rho_{0}$ is the adjoint representation.

If $a, a^{\prime} \in \mathcal{A}_{\text {flat }}(P)$, then there is a gauge transformation $u$ such that $u^{*} a$ and $a^{\prime}$ lie in the same component of $\mathcal{A}_{\text {flat }}(P)$. Moreover, two flat connections a, $a^{\prime}$ lie in the same component of $\mathcal{A}_{\text {flat }}(P)$ if and only if $\mathcal{C S}(a)=\mathcal{C S}\left(a^{\prime}\right)$.

Our proof also identifies precisely when flat connections on $P$ exist. To state this, consider the commutator subgroup $[G, G] \subseteq G$. Then the quotient $P /[G, G]$ is a torus bundle over $Y$. For example, if $G$ is semisimple then $P /[G, G]=Y$, and if $G=\mathrm{U}(r)$ then this quotient is the determinant $U(1)$-bundle. The next result follows from the proof of Proposition 3.3 below.

Corollary 1.3. Let $P \rightarrow Y$ be as in Theorem 1.1. The space $\mathcal{A}_{\text {flat }}(P)$ of flat connections is nonempty if and only if (i) the restriction $P /\left.[G, G]\right|_{\partial H}$ is the trivial bundle, and (ii) for any spherical component $S^{2} \subseteq \partial H$, the restriction $\left.P\right|_{S^{2}}$ is the trivial bundle.

The author's primary interest in Theorem 1.1 is due to its implications for the instanton energy values on certain noncompact 4-manifolds; see [Duncan 2013b]. These 4-manifolds are those of the form $\mathbb{R} \times H^{\infty}$, where

$$
H^{\infty}:=H \cup_{\partial H}([0, \infty) \times \partial H)
$$

is obtained from a Riemannian 3-manifold $H$ by attaching a cylindrical end on its boundary. Given a principal $G$-bundle $P \rightarrow H$, define $P^{\infty} \rightarrow H^{\infty}$ similarly. Then the "manifold at infinity" of $\mathbb{R} \times H^{\infty}$ is the double of $H$ (see Section $3 \mathrm{C}$ ).

Corollary 1.4. Suppose $G$ is a compact, connected Lie group and $H$ is a compact, oriented 3-manifold with boundary. Let $A$ be any finite-energy instanton on $\mathbb{R} \times$ $P^{\infty} \rightarrow \mathbb{R} \times H^{\infty}$, with the instanton equation defined using the product metric. Then there is a flat connection $a_{b}$ on $\bar{H} \cup_{\partial H} H$ such that the energy of $A$ is $\mathcal{C S}_{a}\left(a_{b}\right)$.

Note that the assumptions on $G$ and $H$ are very general. Corollary 1.4 is proved in Section 3C using an extension of a standard argument; see [Taubes 1982; Dostoglou and Salamon 1994; Salamon 1995; Wehrheim 2006; 2005; Nishinou 2010]. See also [Yeung 1991; Etesi 2013] for similar results on instanton energies and characteristic numbers for noncompact manifolds.

\section{Background}

Given a vector bundle $E \rightarrow X$, we will write $\Omega^{\bullet}(X, E):=\oplus_{k} \Omega^{k}(X, E)$ for the space of differential forms on $X$ with values in $E$. We use the wedge product given by $\mu \wedge v=\mu \otimes v-v \otimes \mu$ for real-valued 1-forms $\mu, v$. 
Let $G$ be a compact Lie group, and $\rho_{0}: G \rightarrow \mathrm{U}(W)$ the faithful unitary representation from the introduction. Then define a bilinear form $\langle\cdot, \cdot\rangle$ on the Lie algebra $\mathfrak{g}$ by setting

$$
\langle\mu, \nu\rangle:=-\frac{1}{2 \pi^{2}} \operatorname{Tr}\left(\left(\rho_{0}\right)_{*} \mu \cdot\left(\rho_{0}\right)_{*} \nu\right), \quad \forall \mu, v \in \mathfrak{g},
$$

where the trace is the one on $\mathfrak{u}(W)$. (The normalizing factor $1 / 2 \pi^{2}$ is chosen so that the quantities (4) and (6) below are integers. If $\rho_{0}$ has image in $\mathrm{SU}(W)$ then the more familiar $1 / 4 \pi^{2}$ can be used.) Since we have assumed $\rho_{0}$ is faithful, it follows that $\langle\cdot, \cdot\rangle$ is nondegenerate, and so this defines an Ad-invariant inner product on $\mathfrak{g}$.

Suppose $\pi: P \rightarrow X$ is a principal $G$-bundle over a smooth $n$-manifold $X$; we assume $G$ acts on $P$ on the right. Given a right action $\rho: G \rightarrow \operatorname{Diff}(F)$ of $G$ on a manifold $F$ we will denote the associated bundle by $P \times_{G} F:=(P \times F) / G$. If $F=V$ is a vector space and $G \rightarrow \operatorname{Diff}(V)$ has image in $\operatorname{GL}(V) \subset \operatorname{Diff}(V)$, then $P \times_{G} V$ is a vector bundle and we will write $P(V):=P \times_{G} V$. Pullback by $\pi$ induces an injection

$$
\pi^{*}: \Omega^{\bullet}(X, P(V)) \hookrightarrow \Omega^{\bullet}(P, P \times V)
$$

with image the space of forms that are equivariant and horizontal.

We will write $P(\mathfrak{g})$ for the adjoint bundle associated to the adjoint representation $G \rightarrow \operatorname{GL}(\mathfrak{g})$. The Lie bracket $[\cdot, \cdot]$ on $\mathfrak{g}$ is Ad-invariant, and so this combines with the wedge to define a bilinear map $\mu \otimes v \mapsto[\mu \wedge v]$ on $\Omega^{\bullet}(X, P(\mathfrak{g}))$, endowing $\Omega^{\bullet}(X, P(\mathfrak{g}))$ with the structure of a graded algebra. Similarly, the Ad-invariance of the inner product $\langle\cdot, \cdot\rangle$ implies that it induces a fiberwise inner product on the vector bundle $P(\mathfrak{g})$. This combines with the wedge to give a graded bilinear map

$$
\Omega^{k}(X, P(\mathfrak{g})) \otimes \Omega^{l}(X, P(\mathfrak{g})) \rightarrow \Omega^{k+l}(X), \quad \mu \otimes v \mapsto\langle\mu \wedge v\rangle .
$$

2A. Gauge theory. We denote by $\mathcal{A}(P)$ the set of all connections on $P$. By definition, $\mathcal{A}(P)$ consists of the elements of $\Omega^{1}(P, P \times \mathfrak{g})$ that are both $G$-equivariant and vertical. It follows that $\mathcal{A}(P)$ is an affine space modeled on $\pi^{*} \Omega^{1}(X, P(\mathfrak{g})) \cong$ $\Omega^{1}(X, P(\mathfrak{g}))$. We will write $\mathcal{A}^{1}(P)$ for the completion of $\mathcal{A}(P)$ with respect to the $H^{1}$-Sobolev norm; we will always assume $\mathcal{A}^{1}(P)$ is equipped with the $H^{1}$-topology. The space $\mathcal{A}^{1}(P)$ is well-defined when $X$ is compact; when $X$ is noncompact the $H^{1}$-norm depends on the choice of a smooth reference connection at infinity.

Given any representation $\rho: G \rightarrow \operatorname{GL}(V)$, each connection $A \in \mathcal{A}(P)$ determines a covariant derivative

$d_{A, \rho}: \Omega^{\bullet}(X, P(V)) \rightarrow \Omega^{\bullet+1}(X, P(V)), \quad \mu \mapsto\left(\pi^{*}\right)^{-1}\left(d\left(\pi^{*} \mu\right)+\rho_{*}(A) \wedge \pi^{*} \mu\right)$,

where $d$ is the trivial connection on $P \times V$. When considering the adjoint representation, we will write $d_{A}:=d_{A, \mathrm{Ad}}$. The curvature endomorphism $\operatorname{curv}\left(d_{A, \rho}\right) \in$ 
$\Omega^{2}(X, \operatorname{End}(P(V)))$ is defined by the relation

$$
d_{A, \rho} \circ d_{A, \rho} \mu=\operatorname{curv}\left(d_{A, \rho}\right) \wedge \mu
$$

for all $\mu \in \Omega^{\bullet}(X, P(V))$. We define the curvature (2-form) of $A$ by

$$
F_{A}=\left(\pi^{*}\right)^{-1}\left(d A+\frac{1}{2}[A \wedge A]\right) \in \Omega^{2}(X, P(\mathfrak{g})) .
$$

The curvature 2-form $F_{A}$ recovers the curvature endomorphism $\operatorname{curv}\left(d_{A, \rho}\right)$ in any representation $\rho$ in the sense that

$$
\rho_{*} F_{A}=\operatorname{curv}\left(d_{A, \rho}\right) .
$$

Taking $\rho=$ Ad, we therefore have $\operatorname{curv}\left(d_{A}\right) \wedge \mu=\left[F_{A} \wedge \mu\right]$ for all $\mu \in \Omega^{\bullet}(X, P(\mathfrak{g}))$. Given any $A \in \mathcal{A}(P)$, the covariant derivative and curvature satisfy

$$
d_{A+\mu}=d_{A}+[\mu \wedge \cdot], \quad F_{A+\mu}=F_{A}+d_{A} \mu+\frac{1}{2}[\mu \wedge \mu],
$$

for all $\mu \in \Omega^{1}(X, P(\mathfrak{g}))$. We also have the Bianchi identity $d_{A} F_{A}=0$. A connection $A$ is flat if $F_{A}=0$, and we denote the set of all smooth (resp. $H^{1}$ ) flat connections on $P$ by $\mathcal{A}_{\text {flat }}(P)$ (resp. $\left.\mathcal{A}_{\text {flat }}^{1}(P)\right)$.

Suppose $X$ is a closed, oriented 4-manifold. Then associated to the fixed representation $\rho_{0}: G \rightarrow \mathrm{U}(W)$ from the introduction, we obtain a complex vector bundle $P(W)$ equipped with a Hermitian inner product. In particular, this has well-defined Chern classes $c_{i}:=c_{i}(P(W)) \in H^{2 i}(X, \mathbb{Z})$. The usual Chern-Weil formula says $\kappa(P)=\kappa\left(P ; \rho_{0}\right):=\left(c_{1}^{2}-2 c_{2}\right)[X]=-\frac{1}{4 \pi^{2}} \int_{X} \operatorname{Tr}\left(\operatorname{curv}\left(d_{A, \rho_{0}}\right) \wedge \operatorname{curv}\left(d_{A, \rho_{0}}\right)\right) \in \mathbb{Z}$, for any connection $A \in \mathcal{A}(P)$; the Bianchi identity shows this is independent of the choice of $A$. Here $\operatorname{Tr}(\mu \wedge v)$ is obtained by combining the wedge with the trace on $\mathfrak{u}(W)$. Then equations (2) and (3) show

$$
\kappa(P)=\frac{1}{2} \int_{X}\left\langle F_{A} \wedge F_{A}\right\rangle .
$$

Remark 2.1. This characteristic number can be equivalently defined as follows. Let $B \mathrm{U}(W)$ be the classifying space for the unitary group, and let $\kappa \in H^{4}(B \mathrm{U}(W), \mathbb{Z})$ be given by the square of the first Chern class minus two times the second Chern class. Then $\kappa(P) \in H^{4}(X, \mathbb{Z}) \cong \mathbb{Z}$ is obtained by pulling back $\kappa$ under the map $X \rightarrow B G \rightarrow B \mathrm{U}(W)$; here the first arrow is the classifying map for $P$, and the second is induced by the representation $\rho_{0}: G \rightarrow \mathrm{U}(W)$.

It follows immediately from the definition that $\kappa(P)$ is even if the mod-2 reduction of $c_{1}$ vanishes. Now suppose $\rho_{0}$ is obtained by complexifying a (real) orthogonal representation $G \rightarrow \mathrm{O}(V)$. Then $P(W)=P(V)_{\mathbb{C}}$ is the complexification of the real vector bundle $P(V)$ and so $c_{1}=0$ vanishes. If, in addition, $X=S^{1} \times Y$ is a product, 
then a characteristic class argument shows that $c_{2}$ is even (e.g., see [Duncan 2013a, Section 4.3]), and so $\kappa(P)$ is a multiple of 4 .

For example, consider the case where $G=\mathrm{SO}(r)$ with $r \geq 2$, and $\rho_{0}=\operatorname{Ad}_{\mathbb{C}}$ is the complexified adjoint representation. Then $\kappa(P)=2(r-2) p_{1}\left(P\left(\mathbb{R}^{r}\right)\right)[X]$, where $p_{1}\left(P\left(\mathbb{R}^{4}\right)\right)$ is the Pontryagin class of the vector bundle associated to the standard representation of $\mathrm{SO}(r)$.

As a second example, consider $G=\mathrm{SU}(r)$. Then the integers $\kappa$ coming from the complexified adjoint and standard representations are related by

$$
\kappa\left(P ; \operatorname{Ad}_{\mathbb{C}}\right)=2 r \kappa(P ; \text { standard }) .
$$

A gauge transformation on $P$ is a $G$-equivariant bundle map $P \rightarrow P$ covering the identity. The set $\mathcal{G}(P)$ of gauge transformations on $P$ forms a group, called the gauge group. One may equivalently view the gauge group as the set of $G$ equivariant maps $P \rightarrow G$. Here $G$ acts on itself by conjugation of the inverse, making it a right action. A third equivalent way to view $\mathcal{G}(P)$ is as the space of sections of the bundle $P \times{ }_{G} G \rightarrow X$, where $P \times{ }_{G} G$ is formed using the same action of $G$ on itself.

Denote by $\mathcal{G}_{0}=\mathcal{G}_{0}(P)$ the connected component of the identity in $\mathcal{G}(P)$. We need to specify a topology on $\mathcal{G}(P)$ for the term "connected component" to be meaningful, and we do this by viewing $\mathcal{G}(P)$ as a subspace of the space of functions $P \rightarrow G$, equipped with the $H^{2}$-topology (however, any other Hölder or Sobolev topology would determine the same connected components). We denote by $\mathcal{G}^{2}(P)$ the completion of $\mathcal{G}(P)$ in the $H^{2}$-topology. Note that this depends on a choice of faithful representation of $G$ (see [Wehrheim 2004, Appendix B]), and we take $\rho_{0}$ for this choice.

The gauge group acts on $\Omega^{\bullet}(P, P \times \mathfrak{g})$ and $\mathcal{A}(P) \subset \Omega^{\bullet}(P, P \times \mathfrak{g})$ by pullback. When the dimension of $X$ is three or less, this action is smooth with the specified topologies [Wehrheim 2004, Appendix A]. We note that the action of a gauge transformation $u$ on a connection $A$ can be expressed as

$$
u^{*} A=u^{-1} A u+u^{-1} d u,
$$

where the concatenation on the right is matrix multiplication and $d u$ is the linearization of $u: P \rightarrow G$. In dimensions three or less, Equation (5) combines with the Sobolev multiplication theorem to show that if $u, A$ and $u^{*} A$ are all of Sobolev class $H^{1}$, then $u$ is actually of Sobolev class $H^{2}$.

The group $\mathcal{G}(P)$ also acts on $\Omega^{\bullet}(X, P(\mathfrak{g}))$ by the pointwise adjoint action $(\xi, u) \mapsto \operatorname{Ad}\left(u^{-1}\right) \xi$. In particular, the curvature of $A \in \mathcal{A}(P)$ transforms under $u \in \mathcal{G}(P)$ by

$$
F_{u^{*} A}=\operatorname{Ad}\left(u^{-1}\right) F_{A} .
$$


We introduce a notation convention that is convenient when the dimension of the underlying space $X$ is relevant. If $\operatorname{dim} X=4$, then we use $A, U$ for connections and gauge transformations; if $\operatorname{dim} X=3$, then we use $a, u$ for connections and gauge transformations; if $\operatorname{dim} X=2$, then we use $\alpha, \mu$ for connections and gauge transformations. For example, this provides an effective way to distinguish between a path of gauge transformations $\mu: I \rightarrow \mathcal{G}(P)$ on a surface $X$, and its associated gauge transformation $u \in \mathcal{G}(I \times P)$ on the 3-manifold $I \times X$ defined by $\left.u\right|_{\{t\} \times P}=$ $\mu(t)$.

2B. The Chern-Simons functional. Fix a closed, connected, oriented 3-manifold $Y$, as well as a principal $G$-bundle $P \rightarrow Y$. The space of connections admits a natural 1-form $\lambda \in \Omega^{1}(\mathcal{A}(P), \mathbb{R})$ defined at $a \in \mathcal{A}(P)$ by

$$
\lambda_{a}: T_{a} \mathcal{A}(P) \rightarrow \mathbb{R}, \quad v \mapsto \int_{Y}\left\langle v \wedge F_{a}\right\rangle
$$

The Bianchi identity shows that this is a closed 1-form. Since $\mathcal{A}(P)$ is contractible it follows that $\lambda$ is exact. Fixing a reference connection $a_{0}$, this exact 1-form can therefore be integrated along paths from $a_{0}$ to obtain a real-valued function $\mathcal{C S}_{a_{0}}: \mathcal{A}(P) \rightarrow \mathbb{R}$. One can compute that $\mathcal{C S}_{a_{0}}$ is given by the formula

$$
\mathcal{C S}_{a_{0}}(a):=\int_{Y}\left\langle F_{a_{0}} \wedge v\right\rangle+\frac{1}{2}\left\langle d_{a_{0}} v \wedge v\right\rangle+\frac{1}{6}\langle[v \wedge v] \wedge v\rangle,
$$

where we have set $v:=a-a_{0} \in \Omega^{1}(Y, P(\mathfrak{g}))$. We will typically choose $a_{0}$ to be flat, but this is not always convenient. In general, however, changing $a_{0}$ changes $\mathcal{C S}_{a_{0}}$ by a constant. Projecting $\mathcal{C} \mathcal{S}_{a_{0}}$ to the circle $\mathbb{R} / \mathbb{Z}$, one obtains the Chern-Simons function $\mathcal{C S}: \mathcal{A}(P) \rightarrow \mathbb{R} / \mathbb{Z}$ from the introduction; we will refer to the lift $\mathcal{C} \mathcal{S}_{a_{0}}$ as the Chern-Simons functional. Moreover, $\mathcal{C S}_{a_{0}}$ has a smooth extension from the smooth connections $\mathcal{A}(P)$ to the $H^{1}$-completion $\mathcal{A}^{1}(P)$.

Suppose $a, a^{\prime} \in \mathcal{A}(P)$. Any path $a(\cdot):[0,1] \rightarrow \mathcal{A}(P)$ from $a$ to $a^{\prime}$ can be interpreted as a connection $A$ on $[0,1] \times P \rightarrow[0,1] \times Y$ by requiring that it restricts to $a(t)$ on $\{t\} \times Y$. It follows from the definitions that

$$
\mathcal{C S}_{a_{0}}\left(a^{\prime}\right)-\mathcal{C S}_{a_{0}}(a)=\frac{1}{2} \int_{I \times Y}\left\langle F_{A} \wedge F_{A}\right\rangle .
$$

In the special case where $a^{\prime}=u^{*} a$, with $u \in \mathcal{G}(P)$, the connection $A$ descends to a connection on the mapping torus

$$
P_{u}:=I \times P /(0, u(q)) \sim(1, q),
$$

which is a bundle over $S^{1} \times Y$. Then the above gives

$$
\mathcal{C S}_{a_{0}}\left(u^{*} a\right)-\mathcal{C S}_{a_{0}}(a)=\frac{1}{2} \int_{S^{1} \times Y}\left\langle F_{A} \wedge F_{A}\right\rangle=\kappa\left(P_{u}\right) \in \mathbb{Z}
$$


where we used (4) in the second equality. It follows that the value of this depends only on the path component of $u$ in $\mathcal{G}(P)$. Equation (6) also shows that $\mathcal{C S}_{a_{0}}$ is invariant under the subgroup of gauge transformations $u$ with $\kappa\left(P_{u}\right)=0$ (the 'degree zero' gauge transformations), and that the circle-valued function $\mathcal{C S}: \mathcal{A}(P) \rightarrow \mathbb{R} / \mathbb{Z}$ is invariant under the full gauge group $\mathcal{G}(P)$.

Remark 2.2. The discussion following Equation (4) shows that if the mod-2 reduction of $c_{1}\left(P_{u}(W)\right)$ vanishes, then (6) is even. Similarly, if the fixed representation $\rho_{0}$ is the complexification of a real representation, then (6) is a multiple of 4 .

For completeness we show that the space of flat connections on $P$ is locally path-connected. This implies, for example, that the Chern-Simons critical values are always isolated since the moduli space $\mathcal{A}_{\text {flat }}(P) / \mathcal{G}(P)$ is compact and $\mathcal{C S}_{a_{0}}$ is constant on the path components of $\mathcal{A}_{\text {flat }}(P)$.

Proposition 2.3. The space $\mathcal{A}_{\text {flat }}^{1}(P)$ of flat connections is locally path-connected. In particular, the path components are the connected components.

Proof. Råde [1992] used the heat flow associated to the Yang-Mills equations to show that there is some $\epsilon_{P}>0$ such that if $a \in \mathcal{A}^{1}(P)$ is a connection with $\left\|F_{a}\right\|_{L^{2}} \leq \epsilon_{P}$, then there is a nearby flat connection

$$
\text { Heat }(a) \in \mathcal{A}_{\text {flat }}^{1}(P) \text {. }
$$

Råde shows that the map $a \mapsto \operatorname{Heat}(a)$ is continuous, gauge equivariant and restricts to the identity on $\mathcal{A}_{\text {flat }}^{1}(P)$.

Let $a_{0}, a_{1} \in \mathcal{A}_{\text {flat }}^{1}(P)$. We want to show that if $a_{0}$ and $a_{1}$ are close enough (in $\left.H^{1}\right)$, then they are connected by a path in $\mathcal{A}_{\text {flat }}^{1}(P)$. Consider the straight-line path $a(t)=a_{0}+t\left(a_{1}-a_{0}\right)$. Then

$$
F_{a(t)}=t d_{a_{0}}\left(a_{1}-a_{0}\right)+\frac{t^{2}}{2}\left[a_{1}-a_{0} \wedge a_{1}-a_{0}\right]
$$

and so

$$
\left\|F_{a(t)}\right\|_{L^{2}} \leq\left\|d_{a_{0}}\left(a_{1}-a_{0}\right)\right\|_{L^{2}}+\left\|a_{1}-a_{0}\right\|_{L^{4}}^{2} \leq C\left(\left\|a_{1}-a_{0}\right\|_{H^{1}}+\left\|a_{1}-a_{0}\right\|_{H^{1}}^{2}\right),
$$

where we have used the Sobolev embedding $H^{1} \hookrightarrow L^{4}$. Then $a(t)$ is in the realm of Råde's heat flow map for all $t \in[0,1]$, provided $\left\|a_{1}-a_{0}\right\|_{H^{1}}<\min \left\{1, \epsilon_{P} / 2 C\right\}$. When this is the case, $t \mapsto \operatorname{Heat}(a(t)) \in \mathcal{A}_{\text {flat }}^{1}(P)$ is a path from $a_{0}$ to $a_{1}$, as desired.

\section{Chern-Simons values and instantons}

We prove Theorem 1.1 and Corollary 1.4 in Sections 3B and 3C, respectively. We take a TQFT approach to the proof of Theorem 1.1 in the sense that we treat each connection on $Y=\bar{H} \cup_{\partial} H$ as a pair of connections on $H$ that agree on the boundary. 
This reduces the problem to a study of the flat connections on $H$ and $\partial H$, which is the content of Section 3A.

3A. The components of the gauge group and the space of flat connections. In this section we fix a principal $G$-bundle $P \rightarrow X$, where $X$ is a manifold with (possibly empty) boundary. Unfortunately, the action of the gauge group is rarely free. To account for this, it is convenient to consider the based gauge group $\mathcal{G}_{p}=\mathcal{G}_{p}(P)$ defined as the kernel of the map $\mathcal{G}(P) \rightarrow G$ given by evaluating $u: P \rightarrow G$ at some fixed point $p \in P$. If $X$ is connected, then $\mathcal{G}_{p}$ acts freely on $\mathcal{A}(P)$ (in general, the stabilizer in $\mathcal{G}(P)$ of a connection $A \in \mathcal{A}(P)$ can be identified with the image in $G$ of the evaluation map $u \rightarrow u(p)$ ).

Let $\widetilde{G} \rightarrow G$ be the universal cover. We will be interested in the subgroup $\mathcal{H}=\mathcal{H}(P)$ of gauge transformations $u: P \rightarrow G$ that lift to $G$-equivariant maps $\tilde{u}: P \rightarrow \widetilde{G}$, where the (right) action of $G$ on $\widetilde{G}$ is induced by the conjugation action of $\widetilde{G}$ on itself.

Lemma 3.1. The subgroup $\mathcal{H}$ is a union of connected components of $\mathcal{G}(P)$. In particular, $\mathcal{H}$ contains the identity component $\mathcal{G}_{0}$ of $\mathcal{G}(P)$.

Proof. Consider the aforementioned right action of $G$ on $\widetilde{G}$. Use this action to define a bundle $P \times{ }_{G} \widetilde{G} \rightarrow X$, and consider the natural projection $P \times{ }_{G} \widetilde{G} \rightarrow P \times_{G} G$. Viewing a gauge transformation $u$ as a section of $P \times{ }_{G} G \rightarrow X$, the defining condition of $\mathcal{H}$ is equivalent to the existence of a section $\tilde{u}: X \rightarrow P \times_{G} \widetilde{G}$ lifting $u$. It follows from the homotopy lifting property for the covering space $P \times{ }_{G} \widetilde{G} \rightarrow P \times{ }_{G} G$ that $u$ is an element of $\mathcal{H}$ if and only if $u$ can be connected by a path to an element of $\mathcal{H}$.

Lemma 3.2. Suppose $G$ is compact and connected, and that $X$ has the homotopy type of a connected 2-dimensional $C W$ complex. Then $\mathcal{H} \cap \mathcal{G}_{p}$ is connected, and the inclusion $\mathcal{G}_{p} \subseteq \mathcal{G}(P)$ induces a bijection $\pi_{0}\left(\mathcal{G}_{p}\right) \cong \pi_{0}(\mathcal{G}(P))$. Consequently, $\mathcal{H}$ is the identity component $\mathcal{G}_{0}$ of $\mathcal{G}(P)$.

Proof. First we show that $\mathcal{H} \cap \mathcal{G}_{p}$ is connected. For $u \in \mathcal{H}$, let $\tilde{u}$ be a lift as above. Note that if $u \in \mathcal{G}_{p}$, then $\tilde{u}(p) \in Z(\widetilde{G})$ is in the center and so $\tilde{u}(p)^{-1} \tilde{u}$ is another equivariant lift of $u$. In particular, by replacing $\tilde{u}$ with $\tilde{u}(p)^{-1} \tilde{u}$, we may assume $\tilde{u}$ has been chosen so that $\tilde{u}(p)=e \in \widetilde{G}$. Moreover, by homotoping $u$ we may assume that $u$ (hence $\tilde{u}$ ) restricts to the identity on $\pi^{-1}(B)$, where $B \subset X$ is some open coordinate ball around $x=\pi(p)$. The topological assumptions imply that $B$ can be chosen so the complement $X-B$ deformation retracts to its 1 -skeleton. Since $G$ is connected, the restriction $\left.P\right|_{X-B} \rightarrow X-B$ is trivializable. By equivariance, we may therefore view $\tilde{u}$ simply as a map

$$
\tilde{u}:(X-B, \partial B) \rightarrow(\widetilde{G}, e) .
$$


Now we show $\pi_{0}\left(\mathcal{G}_{p}\right) \cong \pi_{0}(\mathcal{G}(P))$. We may homotope any gauge transformation $u: P \rightarrow G$ so that it is constant on $\pi^{-1}(B) \subset P$, with $B$ as above. Just as above $\left.P\right|_{\bar{B}} \rightarrow \bar{B}$ is the trivial bundle, so gauge transformations on $\left.P\right|_{\bar{B}}$ are exactly maps $\bar{B} \rightarrow G$. Since $G$ is connected, we can obviously find a homotopy rel $\partial B$ of $u:(\bar{B}, \partial B) \rightarrow(G, u(p))$ to a map that sends $x \in B$ to the identity. This shows that $u$ can be homotoped to an element of $\mathcal{G}_{p}$.

Finally, by Lemma 3.1 we have $\mathcal{G}_{0} \subseteq \mathcal{H}$, while the reverse inclusion follows from the conclusions of the previous two paragraphs.

Fix $x \in X$ as well as a point $p \in P$ over $x$. It is well-known that the holonomy provides a map hol : $\mathcal{A}_{\text {flat }}(P) \rightarrow \operatorname{hom}\left(\pi_{1}(X, x), G\right)$. This intertwines the action of $\mathcal{G}(P)$ on $\mathcal{A}_{\text {flat }}(P)$ with the conjugation action of $G$ on itself in the sense that if $\gamma:\left(S^{1}, 1\right) \rightarrow(X, x)$ is a smooth loop, then

$$
\operatorname{hol}_{u^{*} A}(\gamma)=u(p)^{-1} \operatorname{hol}_{A}(\gamma) u(p)
$$

for all gauge transformations $u \in \mathcal{G}(P)$ and flat connections $A$; see [Kobayashi and Nomizu 1963, Proposition 4.1] and [Atiyah and Bott 1983]. Moreover, the holonomy descends to a topological embedding

$$
\mathcal{A}_{\text {flat }}(P) / \mathcal{G}_{p} \hookrightarrow \operatorname{hom}\left(\pi_{1}(X, x), G\right)
$$

with image a union of connected components that are determined by the topological type of the bundle $P$. To determine this set of image components for a given bundle $P$, it is useful to consider the following variation dating back to Atiyah and Bott [1983]. Let $j: G \rightarrow P$ denote the embedding $g \mapsto p \cdot g^{-1}$ (recall $G$ acts on $P$ on the right), and let $j_{*}$ denote the induced map on $\pi_{1}$. Consider the universal cover $\widetilde{G} \rightarrow G$ and denote by $\iota: \pi_{1}(G) \hookrightarrow Z(\widetilde{G})$ the natural inclusion into the center of $\widetilde{G}$. Then there is a homeomorphism

$$
\mathcal{A}_{\text {flat }}(P) /\left(\mathcal{H} \cap \mathcal{G}_{p}\right) \cong\left\{\rho \in \operatorname{hom}\left(\pi_{1}(P, p), \widetilde{G}\right) \mid \rho \circ j_{*}=\iota\right\} .
$$

We defer a proof of (7) until the end of this section.

Proposition 3.3. Assume $G$ is compact and connected. Suppose $X$ is either a closed, connected, oriented surface, or $X=H$ is a compression body. Then the space of flat connections $\mathcal{A}_{\mathrm{flat}}(P)$ is connected when it is nonempty.

Proof. By Lemma 3.2, the group $\mathcal{H} \cap \mathcal{G}_{p}=\mathcal{G}_{0} \cap \mathcal{G}_{p}$ is connected. Moreover, it acts freely on $\mathcal{A}_{\text {flat }}(P)$ since this is the case with $\mathcal{G}_{p}$. We will show the space on the right-hand side of (7) is connected. The proposition follows immediately by the homotopy exact sequence for the bundle $\mathcal{A}_{\text {flat }}(P) \rightarrow \mathcal{A}_{\text {flat }}(P) /\left(\mathcal{H} \cap \mathcal{G}_{p}\right)$.

First assume $X$ is a surface of genus $g \geq 0$. For $g=0$, the space $\mathcal{A}_{\text {flat }}(P) /\left(\mathcal{G}_{0} \cap \mathcal{G}_{p}\right)$ is either a single point or empty, depending on whether $P$ is trivial or not. We may therefore assume $g \geq 1$. The bundle $P \rightarrow X$ is determined up to bundle 
isomorphism by some $\delta \in \pi_{1}(G) \subset Z(\widetilde{G})$. Since $\widetilde{G}$ is simply-connected, it follows that $\widetilde{G}=G_{1} \times \ldots \times G_{k} \times \mathbb{R}^{l}$ for some simple, connected, simply-connected Lie groups $G_{1}, \ldots, G_{k}$. Write $\delta=\left(\delta_{1}, \ldots, \delta_{k}, r\right)$ according to this decomposition.

Now we compute $\pi_{1}(P, p)$. Let $U$ be the complement in $X$ of a point $y$, and let $V$ be a small disk around $y$. Applying the Seifert-van Kampen theorem to the sets $\left.P\right|_{U},\left.P\right|_{V} \subset P$, one finds a presentation for $\pi_{1}(P, p)$ that consists of generators and relations coming from $\pi_{1}(G)$, as well as additional generators $\alpha_{1}, \beta_{1}, \ldots, \alpha_{g}, \beta_{g}$ subject to the relation

$$
\prod_{j=1}^{g}\left[\alpha_{j}, \beta_{j}\right]=\delta,
$$

as well as further relations asserting that each element of $\left\{\alpha_{i}, \beta_{i}\right\}_{i}$ commutes with each generator coming from $\pi_{1}(G)$. Alternatively, the relation (8) can be viewed as arising when one compares trivializations of $\left.P\right|_{U}$ and $\left.P\right|_{V}$ on the overlap $U \cap V$. It follows that $\mathcal{A}_{\text {flat }}(P) / \mathcal{G}_{0} \cap \mathcal{G}_{p}$ can be identified with the set of tuples $\left(A_{i j}, B_{i j}\right)_{i, j}$, for $1 \leq i \leq k+1$ and $1 \leq j \leq g$, where

(i) $A_{i j}, B_{i j} \in G_{i}$, and $\prod_{j=1}^{g}\left[A_{i j}, B_{i j}\right]=\delta_{i}$ for $1 \leq i \leq k$;

(ii) $A_{k j}, B_{k j} \in \mathbb{R}^{l}$, and $\prod_{j=1}^{g}\left[A_{k j}, B_{k j}\right]=r$.

Since $\mathbb{R}^{l}$ is abelian, the tuples $\left(A_{k j}, B_{k j}\right)_{j}$ appearing in (ii) can only exist if $r=0$. This shows that $\mathcal{A}_{\text {flat }}(P)$ is empty if $r \neq 0$, so we may assume $r=0$. (Note that $r=0$ if and only if the torus bundle $P /[G, G]$, from the introduction, is the trivial bundle.)

For $1 \leq i \leq k$, given any $\delta_{i} \in \widetilde{G}$ it can be shown that (a) there always exist tuples $\left(A_{i j}, B_{i j}\right)_{j} \subset G_{i}^{2 g}$ satisfying $\prod_{j=1}^{g}\left[A_{i j}, B_{i j}\right]=\delta_{i}$, and (b) the set of such $\left(A_{i j}, B_{i j}\right)_{j}$ is always connected; see [Alekseev et al. 1998], [Ramadas et al. 1989, Section 2.1] or [Ho and Liu 2003, Fact 3]. It follows that $\mathcal{A}_{\text {flat }}(P) / \mathcal{G}_{0} \cap \mathcal{G}_{p}$ is a product of connected spaces and is therefore connected. This finishes the proof in the case where $X$ is a surface.

Now suppose $X=H$ is a compression body. Then there is a homotopy equivalence $H \simeq\left(\bigvee_{i=1}^{s} \Sigma_{i}\right) \vee\left(\bigvee_{i=1}^{t} S^{1}\right)$ onto a wedge sum of closed, connected, oriented surfaces $\Sigma_{i}$ and circles; note that the surfaces can be identified with the components of the incoming end $\Sigma_{-} \subset \partial H$. It follows from (7) that $\mathcal{A}_{\text {flat }}(P) / \mathcal{G}_{0} \cap \mathcal{G}_{p}$ is homeomorphic to $\left\{\rho \in \operatorname{hom}\left(\pi_{1}\left(P_{1}\right), \widetilde{G}\right) \mid \rho \circ j_{*}=\iota\right\} \times \ldots \times\left\{\rho \in \operatorname{hom}\left(\pi_{1}\left(P_{s}\right), \widetilde{G}\right) \mid \rho \circ j_{*}=\iota\right\} \times(\widetilde{G})^{t}$, where $P_{i} \rightarrow \Sigma_{i}$ is the restriction of $P$ to the surface $\Sigma_{i} \subset H$. By the previous paragraph this is a product of connected spaces, and so is itself connected. 
Remark 3.4. The above proof shows that, when $H$ is a compression body, restricting to the incoming end $\Sigma_{-} \subset \partial H$ yields a surjective map

$$
\frac{\mathcal{A}_{\text {flat }}(P)}{\mathcal{G}_{0} \cap \mathcal{G}_{p}} \rightarrow \frac{\mathcal{A}_{\text {flat }}\left(P_{1}\right)}{\mathcal{G}_{0}\left(P_{1}\right) \cap \mathcal{G}_{p_{1}}\left(P_{1}\right)} \times \ldots \times \frac{\mathcal{A}_{\text {flat }}\left(P_{s}\right)}{\mathcal{G}_{0}\left(P_{s}\right) \cap \mathcal{G}_{p_{s}}\left(P_{s}\right)}
$$

that is a (trivial) principal $\widetilde{G}^{t}$-bundle. Similarly, restricting to the outgoing end $\Sigma_{+} \subset \partial H$ yields an injection

$$
\frac{\mathcal{A}_{\text {flat }}(P)}{\mathcal{G}_{0} \cap \mathcal{G}_{p}} \hookrightarrow \frac{\mathcal{A}_{\text {flat }}\left(P_{+}\right)}{\mathcal{G}_{0}\left(P_{+}\right) \cap \mathcal{G}_{p_{+}}\left(P_{+}\right)},
$$

where $P_{+} \rightarrow \Sigma_{+}$is the restriction of $P$. In particular, a flat connection on $P \rightarrow H$ is determined uniquely, up to $\mathcal{G}_{0}(P) \cap \mathcal{G}_{p}(P)$, by its value on the boundary component $\Sigma_{+}$, and hence by its value on $\partial H$.

Now we verify (7). This can be viewed as arising from the $\widetilde{G}$-valued holonomy, which we now describe. Let $A \in \mathcal{A}(P)$ be a connection. Given a smooth loop $\gamma: S^{1}=\mathbb{R} / \mathbb{Z} \rightarrow P$, consider the induced loop in the base $\pi \circ \gamma: S^{1} \rightarrow X$. Use this to pull $P$ back to a bundle over the circle $(\pi \circ \gamma)^{*} P \rightarrow S^{1}$. The standard ( $G$-valued) holonomy determines a lift $\operatorname{hol}_{A}(\pi \circ \gamma)$ of the quotient map $[0,1] \hookrightarrow S^{1}=\mathbb{R} / \mathbb{Z}$ :

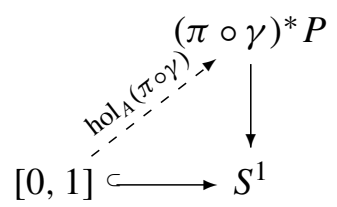

and this lift is unique if we require that it sends 0 to $\gamma(0) \in(\pi \circ \gamma)^{*} P$. On the other hand, $\gamma$ determines a trivialization of this pullback bundle

$$
(\pi \circ \gamma)^{*} P \cong S^{1} \times G, \quad \gamma(t) \mapsto(t, e) .
$$

Compose the lift in (9) with this isomorphism and then with the projection to the $G$-factor in $S^{1} \times G$ to get a map

$$
\operatorname{hol}_{A}(\pi \circ \gamma):[0,1] \rightarrow G
$$

which we denote by the same symbol we used for the standard holonomy. Then the map in (10) sends 0 to the identity $e \in G$ and the value at 1 recovers the standard holonomy for $A$ around $\gamma$. Viewing $\widetilde{G} \rightarrow G$ as a covering space, $\operatorname{hol}_{A}(\pi \circ \gamma)$ lifts to a unique map $\widetilde{\operatorname{hol}_{A}}(\pi \circ \gamma):[0,1] \rightarrow \widetilde{G}$ that sends 0 to $e$. Then we declare the $\widetilde{G}$-valued holonomy of $A$ around $\gamma$ to be the value at 1 :

$$
\operatorname{hol}_{A}^{\widetilde{G}}(\gamma):=\widetilde{\operatorname{hol}}_{A}(\pi \circ \gamma)(1) \in \widetilde{G} .
$$

As with the standard holonomy, one can check that this is multiplicative under concatenation of paths $\gamma$. Similarly, this is equivariant in the following sense. 
Suppose $u \in \mathcal{H}$ and so $u$ lifts to a $G$-equivariant map $\tilde{u}: P \rightarrow \widetilde{G}$. Setting $g:=\tilde{u}(p)$, we have

$$
\operatorname{hol}_{u^{*} A}^{\widetilde{G}}(\gamma)=g^{-1} \operatorname{hol}_{A}^{\widetilde{G}}(\gamma) g
$$

Next, suppose $A$ is a flat connection. Then $\operatorname{hol}_{A}^{\widetilde{G}}(\gamma)$ depends only on the homotopy class of $\gamma$. It follows from the above observations that the $\widetilde{G}$-valued holonomy defines a map $\mathcal{A}_{\text {flat }}(P) \rightarrow \operatorname{hom}\left(\pi_{1}(P, p), \widetilde{G}\right)$, and this intertwines the actions of $\mathcal{H}$ and $\widetilde{G}$. Moreover, from the definition of $\mathcal{G}_{p}$ we have that the $\widetilde{G}$-valued holonomy is invariant under the action of $\mathcal{H} \cap \mathcal{G}_{p}$. We therefore have a well-defined map $\mathcal{A}_{\text {flat }}(P) / \mathcal{H} \cap \mathcal{G}_{p} \rightarrow \operatorname{hom}\left(\pi_{1}(P), \widetilde{G}\right)$. It follows from the definitions above that the image lies in the right-hand side of (7). That this map is a homeomorphism follows from the analogous argument for the standard holonomy, together with the commutativity of the following diagram.

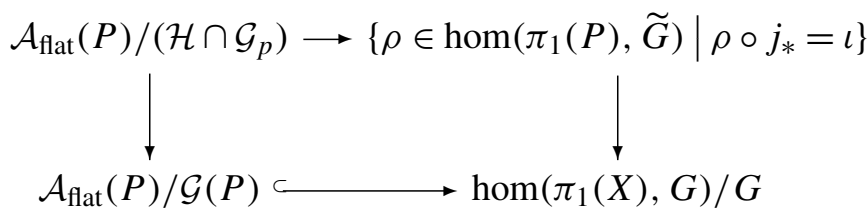

3B. Proof of Theorem 1.1. Write $Y=\bar{H} \cup_{\partial H} H$, where $H$ is a compression body. Fix a collar neighborhood $[0, \epsilon) \times \partial H \hookrightarrow H$ for $\partial H$, and use this to define the smooth structure on $Y$. This smooth structure is independent, up to diffeomorphism, of the choice of collar neighborhood, see [Milnor 1965, Theorem 1.4]. The product structure of this collar neighborhood can be used to define a vector field $v$ on $Y$ that is normal to $\partial H$ and that does not vanish at $\partial H$. Moreover, we assume $v$ has support near $\partial H$, and so $v$ lifts to an equivariant vector field on $P$ that we denote by the same symbol.

Restriction to each of the $H$ factors in $Y=\bar{H} \cup_{\partial H} H$ determines an embedding

$$
\mathcal{A}_{\text {flat }}^{1}(P) \hookrightarrow\left\{(b, c) \in \mathcal{A}_{\text {flat }}^{1}\left(\left.P\right|_{H}\right) \times \mathcal{A}_{\text {flat }}^{1}\left(\left.P\right|_{H}\right) \mid \begin{array}{r}
\left.b\right|_{\partial H}=\left.c\right|_{\partial H}, \\
-\left.\iota_{\nu} b\right|_{\partial H}=\left.\iota_{\nu} c\right|_{\partial H}
\end{array}\right\}
$$

given by

$$
a \mapsto\left(\left.a\right|_{\bar{H}},\left.a\right|_{H}\right) .
$$

A few comments about the defining conditions in the codomain are in order: (i) we are treating $v=\left.v\right|_{H}$ as a vector field on $H$, viewed as the second factor in $\bar{H} \cup_{\partial H} H$; (ii) the negative sign is due to the reversed orientation of the first factor; and (iii) restriction to the hypersurface $\partial H \subset Y$ extends to a bounded linear map $H^{1}(Y) \rightarrow L^{2}(\partial H)$ (see, e.g., [Adams 1975, Theorem 6.3]), and so these equalities should be treated as equalities in the $L^{2}$ sense. 
Suppose $(b, c)$ is in the codomain of (11). These define a connection $a$ on $Y$ by setting $\left.a\right|_{\bar{H}}=b$ and $\left.a\right|_{H}=b$. It is straightforward to check that if $b$ and $c$ are both smooth, then $a$ is continuous and of Sobolev class $H^{1}$ on $Y$. Since the smooth connections are dense in $\mathcal{A}^{1}$, it follows that (11) is surjective, and so we may treat (11) as an identification.

The bijection (11) singles out a preferred subspace that we call the diagonal

$$
\left\{(b, b) \in \mathcal{A}_{\text {flat }}^{1}\left(\left.P\right|_{H}\right) \times \mathcal{A}_{\text {flat }}^{1}\left(\left.P\right|_{H}\right)\left|\iota_{\nu} b\right|_{\partial H}=0\right\} \subset \mathcal{A}_{\text {flat }}^{1}(P) .
$$

It is convenient to consider a slightly larger space $\mathcal{C} \subset \mathcal{A}_{\text {flat }}^{1}(P)$ defined to be the set of flat connections that can be connected by a path to an element of the diagonal (12).

Claim. The diagonal (12) is path-connected. In particular, $\mathcal{C}$ is also path-connected.

To see this, consider diagonal elements $\left(b_{0}, b_{0}\right),\left(b_{1}, b_{1}\right)$. It suffices to prove the claim under the assumption that $b_{0}, b_{1}$ are both smooth and satisfy

$$
\left.\iota_{\nu} b_{0}\right|_{U}=\left.\iota_{\nu} b_{1}\right|_{U}=0
$$

on some neighborhood $U$ of $\partial H$ (this is because the $H^{1}$-completion of the space of these connections recovers (12) and the path-components are stable under completion). By Proposition 3.3 there is a path of flat connections $t \mapsto b_{t} \in \mathcal{A}_{\text {flat }}\left(\left.P\right|_{H}\right)$ connecting $b_{0}$ and $b_{1}$. We will be done if we can ensure that $\left.\iota_{\nu} b_{t}\right|_{\partial H}=0$ for all $t \in[0,1]$. We will accomplish this by putting $b_{t}$ in a suitable ' $v$-temporal gauge', as follows. Restrict attention to the bicollar neighborhood $(-\epsilon, \epsilon) \times \partial H \subset Y$ obtained by doubling the collar neighborhood from the beginning of this section. Let $s$ denote the variable in the $(-\epsilon, \epsilon)$-direction and fix a bump function $\beta$ for $U$ that is equal to 1 on $\partial H$. For each $t \in[0,1]$, define a gauge transformation $u_{t}$ at $(s, h) \in(-\epsilon, \epsilon) \times \partial H$ by the formula

$$
u_{t}(s, h):=\exp \left(-\int_{0}^{s} \iota_{\beta v(\sigma, h)} b_{t}(\sigma, h) d \sigma\right) .
$$

Then $u_{t}$ depends smoothly on all variables, and a computation shows

$$
\iota_{\beta v}\left(u_{t}^{*} b_{t}\right)=0 .
$$

Moreover, it follows from (13) that $u_{t}$ is the identity gauge transformation when $t=0,1$. The claim follows by extending $u_{t}$ to all of $Y$ using a bump function.

It follows from the claim that the Chern-Simons functional is constant on $\mathcal{C}$, since $\mathcal{C} \mathcal{S}_{a_{0}}$ is locally constant on its critical set $\mathcal{A}_{\text {flat }}^{1}(P)$. Suppose Hypothesis 1 holds. We will show that every flat connection in $\mathcal{A}_{\text {flat }}^{1}(P)$ is gauge equivalent to one in $\mathcal{C}$; Theorem 1.1 will then follow immediately from Remark 2.2. In fact, by another density argument, it suffices to show that every smooth flat connection 
is gauge equivalent to one in $\mathcal{C}$. So we fix $a \in \mathcal{A}_{\text {flat }}(P)$. As in the proof of the claim, by applying a suitable gauge transformation, we may assume that $\iota_{\nu} a=0$. Use (11) to identify $a$ with a pair $(b, c) \in \mathcal{A}_{\text {flat }}\left(\left.P\right|_{H}\right) \times \mathcal{A}_{\text {flat }}\left(\left.P\right|_{H}\right)$. Then $b, c$ agree on the boundary, so by Remark 3.4, there is some gauge transformation $u \in \mathcal{G}_{0}\left(\left.P\right|_{H}\right) \cap \mathcal{G}_{p}\left(\left.P\right|_{H}\right)$ for which $u^{*} c=b$. Here we have chosen $p \in H$ to lie in $\Sigma_{+} \subset \partial H$, and we are thinking of the $H$ that appears here as the second factor in $Y=\bar{H} \cup_{\partial H} H$. Our immediate goal is to show that $u$ restricts to the identity gauge transformation on the boundary $\partial H=\Sigma_{+} \cup \Sigma_{-}$. Since $p \in \Sigma_{+}$, it follows that the restriction $\left.u\right|_{\Sigma_{+}}$lies in $\mathcal{G}_{p}\left(\left.P\right|_{\Sigma_{+}}\right)$, which acts freely. Since $b$ and $c$ agree on $\Sigma_{+}$, it must be the case that $\left.u\right|_{\Sigma_{+}}$is the identity. Turning attention to $\Sigma_{-}$, for each component $\Sigma^{\prime} \subset \Sigma_{-}$, the restriction $\left.u\right|_{\Sigma^{\prime}}$ lies in the identity component of the gauge group. In particular, by Hypothesis 1 we have that $\left.u\right|_{\partial H}=e$ is the identity. At this point we have that $u$ is a gauge transformation on $H \subset Y$ that is the identity on all of $\partial H$. Then $u$ extends over $\bar{H} \subset Y$ by the identity to define a continuous gauge transformation $u^{(1)}=(e, u)$ on $P$. This is of Sobolev class $H^{1}$. We also have $\left(u^{(1)}\right)^{*} a \in \mathcal{C}$, since under (11) the connection $\left(u^{(1)}\right)^{*} a$ corresponds to the pair $(b, b)=\left(b, u^{*} c\right)$ and we have assumed $\iota_{\nu} a=0$. Finally, since $u^{(1)}, a$ and $\left(u^{(1)}\right)^{*} a$ are all $H^{1}$, it follows from (5) that $u^{(1)}$ is $H^{2}$. This finishes the proof of Theorem 1.1 under Hypothesis 1.

Remark 3.5. Continue to assume Hypothesis 1, and suppose $a, a^{\prime}$ are flat connections. Then the construction of the previous paragraph shows that there is a gauge transformation $w \in \mathcal{H}(P)$ such that $w^{*} a$ and $a^{\prime}$ lie in the same path component. If we further assume that $\mathcal{C} \mathcal{S}_{a_{0}}(a)=\mathcal{C} \mathcal{S}_{a_{0}}\left(a^{\prime}\right)$, then it follows that $\kappa\left(P_{w}\right)=0$. In many cases, if $w \in \mathcal{H}$ and $\kappa\left(P_{w}\right)=0$, then $w$ necessarily lies in the identity component. For example, this is well-known when $G=\mathrm{U}(r)$ or $\mathrm{SU}(r)$ and $\rho_{0}: G \rightarrow \mathrm{U}\left(\mathbb{C}^{r}\right)$ is the standard representation [Freed and Uhlenbeck 1991, page 79], or if $G=\mathrm{PU}(r)$ and $\rho_{0}$ is the adjoint representation [Duncan 2013a]. In such cases, it follows that $a$ and $a^{\prime}$ lie in the same component of $\mathcal{A}_{\text {flat }}(P)$.

To prove the theorem without Hypothesis 1, we follow a strategy of Wehrheim [2006]. Let $n_{G}$ be as in the definition of $N_{G}$. Without Hypothesis 1 it may not be the case that $u \in \mathcal{G}\left(\left.P\right|_{H}\right)$ restricts to the identity on $\Sigma_{-}$. Write $\Sigma_{-}=\Sigma_{1} \cup \cdots \cup \Sigma_{S}$ in terms of its connected components and write $P_{i}$ for the restriction of $P$ to $\Sigma_{i} \subset \partial H$. Since $G$ is compact, the stabilizer subgroup in $\mathcal{G}\left(P_{i}\right)$ of each restriction $\left.b\right|_{\Sigma_{i}}$ has only finitely many components, and so there is some integer $n \leq n_{G}$ for which $\left.u^{n}\right|_{\Sigma_{i}}$ lies in the identity component of the stabilizer group for $\left.b\right|_{\Sigma_{i}}$. For simplicity we assume $\left.u^{n}\right|_{\Sigma_{i}}=e$ is the identity for each $i$; one can check that the following argument can be easily reduced to this case.

View $H$ as a cobordism from $\Sigma_{-}$to $\Sigma_{+}$(we may assume $\Sigma_{-}$is not empty, otherwise Hypothesis 1 is satisfied), and define a manifold $Y^{(n)}$ by gluing $H$ to 
itself $2 n$ times:

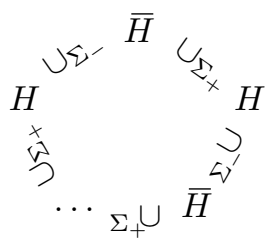

Define a bundle $P^{(n)} \rightarrow Y^{(n)}$ similarly. Then $a=(b, c)$ determines a continuous flat connection on $P^{(n)}$ by the formula

$$
a^{(n)}:=(b, c, b, c, \ldots, b, c) .
$$

The notation means that the $k$-th component lies in the $k$-th copy of $H$ in (14). Similarly, the reference connection $a_{0}$ defines a reference connection $a_{0}^{(n)}$ on $P^{(n)}$, and the gauge transformation $u$ determines a continuous gauge transformation on $P^{(n)}$ by

$$
u^{(n)}:=\left(e, u, u, u^{2}, u^{2}, \ldots, u^{n-1}, u^{n-1}, u^{n}\right) .
$$

Let $\mathcal{C S}^{(n)}$ denote the Chern-Simons functional for $P^{(n)}$ defined using $a_{0}^{(n)}$. Then (6) and the additivity of the integral over its domain give

$$
\mathcal{C S}^{(n)}\left(\left(u^{(n)}\right)^{*} a^{(n)}\right)=\mathcal{C} \mathcal{S}^{(n)}\left(a^{(n)}\right)+\kappa\left(P_{u^{(n)}}\right)=n \mathcal{C} \mathcal{S}_{a_{0}}(a)+\kappa\left(P_{u^{(n)}}\right) .
$$

In addition, the pullback of $a^{(n)}$ by $u^{(n)}$ is $\left(b, b, u^{*} b, u^{*} b, \ldots,\left(u^{n-1}\right)^{*} b,\left(u^{n-1}\right)^{*} b\right)$, and so

$$
\mathcal{C S}^{(n)}\left(\left(u^{(n)}\right)^{*} a^{(n)}\right)=n \mathcal{C S} \mathcal{S}_{a_{0}}\left(a^{\prime}\right)+k_{n}, \quad k_{n}:=\frac{1}{2} n(n-1) \kappa\left(P_{u}\right) \in \mathbb{Z},
$$

where $a^{\prime} \in \mathcal{C}$ is the connection corresponding to $(b, b)$ under (11). Combining these gives $\mathcal{C S}_{a_{0}}(a)-\mathcal{C} \mathcal{S}_{a_{0}}\left(a^{\prime}\right) \in \frac{1}{n} \mathbb{Z} \subseteq \frac{1}{N_{G}} \mathbb{Z}$.

3C. The energies of instantons. Let $P^{\infty} \rightarrow H^{\infty}$ be as in Corollary 1.4, and let $g$ be the cylindrical end metric on $H^{\infty}$. Equip the 4-manifold $\mathbb{R} \times H^{\infty}$ with the product metric, and denote by $Q \rightarrow \mathbb{R} \times H^{\infty}$ the pullback of $P^{\infty}$ under the projection $\mathbb{R} \times H^{\infty} \rightarrow H^{\infty}$. The energy of a connection $A \in \mathcal{A}(Q)$ is defined to be

$$
\frac{1}{2}\left\|F_{A}\right\|_{L^{2}\left(\mathbb{R} \times H^{\infty}\right)}=\frac{1}{2} \int_{\mathbb{R} \times H^{\infty}}\left\langle F_{A} \wedge * F_{A}\right\rangle,
$$

where $*$ is the Hodge star coming from the metric. We will always assume the energy of $A$ is finite. We say that $A$ is an instanton if $* F_{A}= \pm F_{A}$. It follows that the energy of any instanton is given, up to a sign, by

$$
\frac{1}{2} \int_{\mathbb{R} \times H^{\infty}}\left\langle F_{A} \wedge F_{A}\right\rangle
$$

In this section we will prove Corollary 1.4 by showing that (15) is equal to $\mathcal{C S}_{a_{0}}\left(a_{b}\right)$ for some flat connections $a_{b}, a_{0}$ on $Y:=\bar{H} \cup_{\partial H} H$. First we introduce some notation. 
Recalling the decomposition (1), there is a projection

$$
\mathbb{R} \times H^{\infty} \rightarrow \mathbb{H}
$$

to the upper half-plane, sending $\{s\} \times H$ to $(s, 0) \in \mathbb{U}$, and sending each element of $\{(s, t)\} \times \partial H$ to $(s, t)$. (This projection is continuous, but not differentiable.) Note that for each $\tau \in(0, \infty)$, the inverse image under (16) of the semicircle

$$
\{(\tau \cos (\theta), \tau \sin (\theta)) \mid \theta \in[0, \pi]\} \subset \mathbb{M}
$$

is the closed 3-manifold

$$
Y_{\tau}:=\bar{H} \cup_{\{0\} \times \partial H}([0, \tau \pi] \times \partial H) \cup_{\{\tau \pi\} \times \partial H} H .
$$

In the degenerate case $\tau=0$, we declare $Y_{0}$ to be the inverse image under (16) of the origin; so $Y_{0}=\{0\} \times H$. Then we have

$$
\mathbb{R} \times H^{\infty}=\bigcup_{\tau \geq 0} Y_{\tau} .
$$

Moreover, for each $\tau>0$, there is an identification $Y_{\tau} \cong Y_{1}$ induced from the obvious linear map $[0, \tau \pi] \cong[0, \pi]$. This identification is continuous, but when $\tau \neq 1$ this identification is not smooth due to the directions transverse to $\{0, \tau \pi\} \times \partial H$ in $Y_{\tau}$. We note also that we can identify $Y_{1}$ with the double $Y$; however we find it convenient to work with $Y_{1}$ rather than $Y$ at this stage. In summary, we have defined a continuous embedding

$$
\Pi:(0, \infty) \times Y_{1} \rightarrow \mathbb{R} \times H^{\infty}
$$

with image the complement of $Y_{0}$; this map is not smooth. We think of $\Pi$ as providing certain "polar coordinates" on $\mathbb{R} \times H^{\infty}$.

Fix a connection $A$. Then we can write the pullback under $\Pi$ as

$$
\Pi^{*} A=a(\tau)+p(\tau) d \tau,
$$

where $\tau$ is the coordinate on $(0, \infty), a(\cdot)$ is a path of connections on $Y_{1}$, and $p(\cdot)$ is a path of 0 -forms on $Y_{1}$. Fixing $\tau$, the failure of $\Pi$ to be smooth implies that the connection $a(\tau)$ will not be continuous on $Y_{1}$, unless

$$
\iota_{\nu} A=0 .
$$

Here, $v$ is the normal vector to the hypersurface $\mathbb{R} \times \partial H \subset \mathbb{R} \times H^{\infty}$. However, by performing a suitable gauge transformation to $A$, we can always achieve (17). (See the previous section for a similar construction; also note that the action of the gauge group on $A$ does not change the value of (15).) When (17) holds it follows that the connection $a(\tau)$

- is continuous everywhere on $Y_{1}$,

- is smooth away from the hypersurface $\{0, \pi\} \times \partial H \subset Y_{1}$, and

- has bounded derivative near this hypersurface. 
In particular, $a(\tau)$ is of Sobolev class $H^{1}$ on $Y_{1}$.

Now we introduce a convenient reference connection $a_{0}$ on $Y_{1}$ with which we will define $\mathcal{C} \mathcal{S}_{a_{0}}$. This reference connection will depend on the given connection $A$; we continue to assume that (17) holds. Define $a_{0}$ on the first copy of $H$ in $Y_{1}$ by declaring it to equal $\left.A\right|_{Y_{0}}$, where we are identifying $Y_{0}$ with $H$ in the obvious way. Define $a_{0}$ on the second copy of $H$ to also equal $\left.A\right|_{Y_{0}}$. It remains to define $a_{0}$ on the cylinder $[0, \pi] \times \partial H$, and there is a unique way to do this if we require that $a_{0}$ is (i) continuous and (ii) constant in the $[0, \pi]$-direction. It follows from (17) that $a_{0}$ is of Sobolev class $H^{1}$. Moreover,

$$
\lim _{\tau \rightarrow 0^{+}} a(\tau)=a_{0}
$$

where this limit is in the $H^{1}$-topology on $Y_{1}$ (this is basically just the statement that $A$ is continuous at $\left.Y_{0} \subset \mathbb{R} \times H^{\infty}\right)$. Note that this choice of $a_{0}$ may not be flat. However, it turns out that $\mathcal{C} \mathcal{S}_{a_{0}}=\mathcal{C} \mathcal{S}_{a_{1}}$ for some flat connection $a_{1}$ (in fact, any flat connection in the diagonal (12) will do); see Remark 3.6.

Now we prove Corollary 1.4. At this stage the argument follows essentially as in [Wehrheim 2006, Theorem 1.1]; we recall the details for convenience. Let $A$ be any finite energy connection on $\mathbb{R} \times H^{\infty}$, and assume it has been put in a gauge so that (17) holds. Use the identity $F_{\Pi^{*} A}=F_{a}+d \tau \wedge\left(\partial_{\tau} a-d_{a} p\right)$ to get

$$
\frac{1}{2} \Pi^{*}\left\langle F_{A} \wedge F_{A}\right\rangle=d \tau \wedge\left\langle F_{a} \wedge\left(\partial_{\tau} a-d_{a} p\right)\right\rangle .
$$

Integrate both sides and use the fact that the image of $\Pi$ has full measure in $\mathbb{R} \times H^{\infty}$ to get

$$
\begin{aligned}
\frac{1}{2} \int_{\mathbb{R} \times H^{\infty}}\left\langle F_{A} \wedge F_{A}\right\rangle & =\int_{0}^{\infty} \int_{Y_{1}} d \tau \wedge\left\langle F_{a} \wedge \partial_{\tau} a\right\rangle \\
& =\int_{0}^{\infty} \frac{d}{d \tau} \mathcal{C} \mathcal{S}_{a_{0}}(a(\tau)) d \tau \\
& =\lim _{\tau \rightarrow \infty} \mathcal{C} \mathcal{S}_{a_{0}}(a(\tau))-\lim _{\tau \rightarrow 0^{+}} \mathcal{C S}_{a_{0}}(a(\tau)),
\end{aligned}
$$

where we used the Bianchi identity to kill off the $d_{a} p$-term, and then used the definition of $\mathcal{C} \mathcal{S}_{a_{0}}$. From the definition of $a_{0}$, we have

$$
\lim _{\tau \rightarrow 0^{+}} \mathcal{C S}_{a_{0}}(a(\tau))=\mathcal{C S}_{a_{0}}\left(a_{0}\right)=0,
$$

so it suffices to consider the limit at $\infty$.

Notice that (18) shows that $\lim _{\tau \rightarrow \infty} \mathcal{C S}_{a_{0}}(a(\tau))$ exists. The goal now is to show that this limit equals $\mathcal{C S}_{a_{0}}\left(a_{b}\right)$ for some flat connection $a_{b}$. Endow $Y_{1}$ with the metric induced from $d s^{2}+g$ via the inclusion $Y_{1} \subset \mathbb{R} \times H^{\infty}$. Then it follows from 
the definitions that

$$
\int_{1}^{\infty}\left\|F_{a(\tau)}\right\|_{L^{2}\left(Y_{1}\right)}^{2} \leq\left\|F_{A}\right\|_{L^{2}\left(\mathbb{R} \times H^{\infty}\right)}^{2} .
$$

Since the energy of $A$ is finite, the integral over $[1, \infty)$ on the left converges and so there is a sequence $\tau_{i} \in \mathbb{R}$ with

$$
\left\|F_{a\left(\tau_{i}\right)}\right\|_{L^{2}\left(Y_{1}\right)}^{2} \stackrel{i}{\rightarrow} 0 \quad \text { and } \quad \tau_{i} \stackrel{i}{\rightarrow} \infty .
$$

By Uhlenbeck's weak compactness theorem [1982], we can find

- a subsequence of the $\left\{a\left(\tau_{i}\right)\right\}$, denoted by $\left\{a_{i}\right\}$,

- a sequence of gauge transformations $\left\{u_{i}\right\}$, and

- a flat connection $a_{\infty}$,

for which $\left\{u_{i}^{*} a_{i}\right\}$ converges to $a_{\infty}$ weakly in $H^{1}$ and hence strongly in $L^{4}$. This convergence is enough to put each $u_{i}^{*} a_{i}$ in Coulomb gauge with respect to $a_{\infty}$ [Wehrheim 2004, Theorem 8.1], so by redefining each $u_{i}$ we may assume this is the case. Then $u_{i}^{*} a_{i}$ converges to $a_{\infty}$ strongly in $H^{1}$. Since $\mathcal{C} \mathcal{S}_{a_{0}}$ is continuous in the $H^{1}$-topology, we have

$$
\lim _{i \rightarrow \infty} \mathcal{C S}_{a_{0}}\left(u_{i}^{*} a_{i}\right)=\mathcal{C} \mathcal{S}_{a_{0}}\left(a_{\infty}\right)
$$

On the other hand,

$$
\mathcal{C S}_{a_{0}}\left(u_{i}^{*} a_{i}\right)-\mathcal{C S}_{a_{0}}\left(a_{i}\right)=\kappa\left(P_{u_{i}}\right) \in \mathbb{Z}
$$

for all $i$. Since $\mathcal{C S}_{a_{0}}\left(u_{i}^{*} a_{i}\right)$ and $\mathcal{C} \mathcal{S}_{a_{0}}\left(a_{i}\right)$ both converge, it follows that $\kappa\left(P_{u_{i}}\right)$ is constant for all but finitely many $i$. By passing to yet another subsequence, we may assume that $\kappa\left(P_{u_{i}}\right)$ is constant for all $i$. Then there is some gauge transformation $u$ such that $\kappa\left(P_{u}\right)=\kappa\left(P_{u_{i}}\right)$ for all $i$ (just take $u$ to be one of the $\left.u_{i}\right)$. This gives

$$
\begin{aligned}
\frac{1}{2} \int_{\mathbb{R} \times H^{\infty}}\left\langle F_{A} \wedge F_{A}\right\rangle & =\lim _{i \rightarrow \infty} \mathcal{C} \mathcal{S}_{a_{0}}\left(a_{i}\right)=\lim _{i \rightarrow \infty} \mathcal{C} \mathcal{S}_{a_{0}}\left(u_{i}^{*} a_{i}\right)-\kappa\left(P_{u_{i}}\right) \\
& =\mathcal{C} \mathcal{S}_{a_{0}}\left(a_{\infty}\right)-\kappa\left(P_{u}\right)=\mathcal{C} \mathcal{S}_{a_{0}}\left(\left(u^{-1}\right)^{*} a_{\infty}\right) .
\end{aligned}
$$

So taking $a_{b}:=\left(u^{-1}\right)^{*} a_{\infty}$ finishes the proof.

Remark 3.6. Here we address the fact that the reference connection $a_{0}$, constructed in the proof above, may not be a flat connection. We address this from two different angles. First of all, the quantity (15) is independent of the choice of connection $A$, provided that one restricts to connections with the same asymptotic behavior at infinity. In particular, one can always modify the connection $A$ so that its restriction to $Y_{0}$ is flat. This forces $a_{0}$ to be flat.

Secondly, the argument of the previous paragraph suggests that the value $\mathcal{C S}_{a_{0}}(a)$ is somehow independent of $a_{0}$. It is interesting to see this explicitly without 
modifying the original connection $A$. There is an obvious $\mathbb{Z}_{2}$ action on $Y=\bar{H} \cup_{\partial H} H$ given by interchanging the two $H$-factors. Call a form or connection on $Y$ symmetric if it is fixed by this action. For example, all elements of the diagonal (12) are symmetric. The key observation here is that $a_{0}$ is symmetric. Then we claim that function $\mathcal{C S}_{a_{0}}$ is independent of the choice of $a_{0}$ from the class of symmetric connections. Indeed, suppose $a_{1}$ is a second connection that is symmetric. We want to show that $\mathcal{C S}_{a_{0}}(a)=\mathcal{C S}_{a_{1}}(a)$ for all connections $a$. From the definition of the Chern-Simons functional we have

$$
\mathcal{C S}_{a_{0}}(a)-\mathcal{C S}_{a_{1}}(a)=-\mathcal{C S}_{a}\left(a_{0}\right)+\mathcal{C S}_{a}\left(a_{1}\right) .
$$

Note that the right-hand side is actually independent of $a$, since changing the connection $a$ changes $\mathcal{C} \mathcal{S}_{a}$ by a constant. We can therefore replace $a$ with $a_{0}$ on the right-hand side to get

$$
\mathcal{C S}_{a_{0}}(a)-\mathcal{C S}_{a_{1}}(a)=\mathcal{C S}_{a_{0}}\left(a_{1}\right)=\int_{Y}\left\langle F_{a_{0}} \wedge v\right\rangle+\frac{1}{2}\left\langle d_{a_{0}} v \wedge v\right\rangle+\frac{1}{6}\langle[v \wedge v] \wedge v\rangle,
$$

where $v:=a_{1}-a_{0}$. Let $c s_{a_{0}}(v)$ denote the integrand on the right. Now use the following facts: (i) $Y$ decomposes into two copies of $H$, (ii) the two copies of $H$ have opposite orientations, and (iii) $c s_{a_{0}}(v)$ is symmetric (it is made up of the symmetric $\left.a_{0}, a_{1}\right)$. These allow us to compute

$$
\mathcal{C S}_{a_{0}}(a)-\mathcal{C S}_{a_{1}}(a)=\int_{\bar{H}} c s_{a_{0}}(v)+\int_{H} c s_{a_{0}}(v)=-\int_{H} c s_{a_{0}}(v)+\int_{H} c s_{a_{0}}(v)=0 .
$$

\section{Acknowledgments}

Duncan would like to thank Chris Woodward, Sai Kee Yeung, Sushmita Venugopalan, Dan Freed, and Tom Parker for valuable discussions, comments and suggestions. This work was partially supported by NSF Grant DMS 0739208.

\section{References}

[Adams 1975] R. A. Adams, Sobolev spaces, Pure and Applied Mathematics, Academic Press, New York, 1975. MR 56 \#9247 Zbl 0314.46030

[Alekseev et al. 1998] A. Alekseev, A. Malkin, and E. Meinrenken, "Lie group valued moment maps", J. Differential Geom. 48:3 (1998), 445-495. MR 99k:58062 Zbl 0948.53045

[Atiyah and Bott 1983] M. F. Atiyah and R. Bott, "The Yang-Mills equations over Riemann surfaces", Philos. Trans. Roy. Soc. London Ser. A 308:1505 (1983), 523-615. MR 85k:14006 Zbl 0509.14014 [Auckly 1994] D. R. Auckly, "Chern-Simons invariants of 3-manifolds which fiber over $S^{1}$ ", Internat. J. Math. 5:2 (1994), 179-188. MR 95b:57030 Zbl 0854.57012

[Dostoglou and Salamon 1994] S. Dostoglou and D. A. Salamon, "Self-dual instantons and holomorphic curves”, Ann. of Math. (2) 139:3 (1994), 581-640. MR 95g:58050 Zbl 0812.58031

[Duncan 2013a] D. Duncan, "On the components of the gauge group for PU( $r$-bundles", preprint, 2013. arXiv 1311.5611 
[Duncan 2013b] D. L. Duncan, Compactness results for the quilted Atiyah-Floer conjecture, Ph.D. thesis, Rutgers The State University of New Jersey - New Brunswick, 2013, Available at http:// search.proquest.com/docview/1441954671.

[Etesi 2013] G. Etesi, "On the energy spectrum of Yang-Mills instantons over asymptotically locally flat spaces”, Comm. Math. Phys. 322:1 (2013), 1-17. MR 3073155 Zbl 1270.81136

[Freed and Uhlenbeck 1991] D. S. Freed and K. K. Uhlenbeck, Instantons and four-manifolds, 2nd ed., Mathematical Sciences Research Institute Publications 1, Springer, New York, 1991. MR 91i:57019 Zbl 0968.57502

[Ho and Liu 2003] N.-K. Ho and C.-C. M. Liu, "Connected components of the space of surface group representations", Int. Math. Res. Not. 2003:44 (2003), 2359-2372. MR 2004h:53116 Zbl 1043.53064

[Kirk and Klassen 1993] P. Kirk and E. Klassen, "Chern-Simons invariants of 3-manifolds decomposed along tori and the circle bundle over the representation space of $T^{2}$, Comm. Math. Phys. 153:3 (1993), 521-557. MR 94d:57042 Zbl 0789.57011

[Kobayashi and Nomizu 1963] S. Kobayashi and K. Nomizu, Foundations of differential geometry, I, Wiley, New York, 1963. MR 97c:53001a

[Milnor 1965] J. Milnor, Lectures on the h-cobordism theorem, Notes by L. Siebenmann and J. Sondow, Princeton University Press, Princeton, 1965. MR 32 \#352

[Neumann and Yang 1995] W. D. Neumann and J. Yang, "Invariants from triangulations of hyperbolic 3-manifolds", Electron. Res. Announc. Amer. Math. Soc. 1:2 (1995), 72-79. MR 97d:57017 Zbl 0851.57013

[Nishi 1998] H. Nishi, "SU(n)-Chern-Simons invariants of Seifert fibered 3-manifolds", Internat. J. Math. 9:3 (1998), 295-330. MR 99i:57053 Zbl 0911.57010

[Nishinou 2010] T. Nishinou, "Convergence of adiabatic family of anti-self-dual connections on products of Riemann surfaces”, J. Math. Phys. 51:2 (2010), 022306, 10. MR 2011b:53048

[Råde 1992] J. Råde, "On the Yang-Mills heat equation in two and three dimensions", J. Reine Angew. Math. 431 (1992), 123-163. MR 94a:58041 Zbl 0760.58041

[Ramadas et al. 1989] T. R. Ramadas, I. M. Singer, and J. Weitsman, "Some comments on ChernSimons gauge theory”, Comm. Math. Phys. 126:2 (1989), 409-420. MR 90m:58218 Zbl 0686.53066

[Reznikov 1996] A. Reznikov, "Rationality of secondary classes", J. Differential Geom. 43:3 (1996), 674-692. MR 98b:14006 Zbl 0874.32009

[Salamon 1995] D. Salamon, "Lagrangian intersections, 3-manifolds with boundary, and the AtiyahFloer conjecture", pp. 526-536 in Proceedings of the International Congress of Mathematicians (Zürich, 1994), vol. 1, edited by S. D. Chatterji, Birkhäuser, 1995. MR 97e:57040 Zbl 0854.58021

[Taubes 1982] C. H. Taubes, "Self-dual Yang-Mills connections on non-self-dual 4-manifolds", J. Differential Geom. 17:1 (1982), 139-170. MR 83i:53055 Zbl 0484.53026

[Uhlenbeck 1982] K. K. Uhlenbeck, "Connections with $L^{p}$ bounds on curvature", Comm. Math. Phys. 83:1 (1982), 31-42. MR 83e:53035 Zbl 0499.58019

[Wehrheim 2004] K. Wehrheim, Uhlenbeck compactness, EMS Series of Lectures in Mathematics 1, European Mathematical Society, Zürich, 2004. MR 2004m:53045 Zbl 1055.53027

[Wehrheim 2005] K. Wehrheim, "Lagrangian boundary conditions for anti-self-dual instantons and the Atiyah-Floer conjecture", J. Symplectic Geom. 3:4 (2005), 703-747. MR 2007j:53114 Zbl 0559.57001

[Wehrheim 2006] K. Wehrheim, "Energy identity for anti-self-dual instantons on $\mathbb{C} \times \Sigma$ ", Math. Res. Lett. 13:1 (2006), 161-166. MR 2006h:53019 Zbl 1111.53023 
[Wehrheim and Woodward 2009] K. Wehrheim and C. Woodward, "Floer field theory", Preprint, 2009, Available at https://math.berkeley.edu/ katrin/papers/fielda.pdf.

[Yeung 1991] S.-K. Yeung, "Integrality of characteristic numbers on complete Kähler manifolds", Math. Ann. 289:3 (1991), 491-516. MR 92b:32015

Received October 7, 2014. Revised March 25, 2015.

DAVID L. DUNCAN

DEPARTMENT OF MATHEMATICS

MICHIGAN STATE UNIVERSITY

619 RED CEDAR ROAD

EAST LANSING, MI 48824

UNITED STATES

duncan42@math.msu.edu 


\title{
PACIFIC JOURNAL OF MATHEMATICS
}

\author{
msp.org/pjm
}

Founded in 1951 by E. F. Beckenbach (1906-1982) and F. Wolf (1904-1989)

\section{EDITORS}

Don Blasius (Managing Editor)

Department of Mathematics

University of California

Los Angeles, CA 90095-1555

blasius@math.ucla.edu

\author{
Paul Balmer \\ Department of Mathematics \\ University of California \\ Los Angeles, CA 90095-1555 \\ balmer@math.ucla.edu \\ Robert Finn \\ Department of Mathematics \\ Stanford University \\ Stanford, CA 94305-2125 \\ finn@math.stanford.edu \\ Sorin Popa \\ Department of Mathematics \\ University of California \\ Los Angeles, CA 90095-1555 \\ popa@math.ucla.edu
}

\author{
Vyjayanthi Chari \\ Department of Mathematics \\ University of California \\ Riverside, CA 92521-0135 \\ chari@math.ucr.edu \\ Kefeng Liu \\ Department of Mathematics \\ University of California \\ Los Angeles, CA 90095-1555 \\ liu@math.ucla.edu \\ Jie Qing \\ Department of Mathematics \\ University of California \\ Santa Cruz, CA 95064 \\ qing@ cats.ucsc.edu
}

\section{PRODUCTION}

Silvio Levy, Scientific Editor, production@msp.org

\section{SUPPORTING INSTITUTIONS}

ACADEMIA SINICA, TAIPEI

CALIFORNIA INST. OF TECHNOLOGY

INST. DE MATEMÁTICA PURA E APLICADA

KEIO UNIVERSITY

MATH. SCIENCES RESEARCH INSTITUTE

NEW MEXICO STATE UNIV.

OREGON STATE UNIV.

\author{
STANFORD UNIVERSITY \\ UNIV. OF BRITISH COLUMBIA \\ UNIV. OF CALIFORNIA, BERKELEY \\ UNIV. OF CALIFORNIA, DAVIS \\ UNIV. OF CALIFORNIA, LOS ANGELES \\ UNIV. OF CALIFORNIA, RIVERSIDE \\ UNIV. OF CALIFORNIA, SAN DIEGO \\ UNIV. OF CALIF., SANTA BARBARA
}

\author{
Daryl Cooper \\ Department of Mathematics \\ University of California \\ Santa Barbara, CA 93106-3080 \\ cooper@math.ucsb.edu \\ Jiang-Hua Lu \\ Department of Mathematics \\ The University of Hong Kong \\ Pokfulam Rd., Hong Kong \\ jhlu@maths.hku.hk \\ Paul Yang \\ Department of Mathematics \\ Princeton University \\ Princeton NJ 08544-1000 \\ yang@math.princeton.edu
}

These supporting institutions contribute to the cost of publication of this Journal, but they are not owners or publishers and have no responsibility for its contents or policies.

See inside back cover or msp.org/pjm for submission instructions.

The subscription price for 2016 is US $\$ 440 /$ year for the electronic version, and $\$ 600 /$ year for print and electronic.

Subscriptions, requests for back issues and changes of subscribers address should be sent to Pacific Journal of Mathematics, P.O. Box 4163, Berkeley, CA 94704-0163, U.S.A. The Pacific Journal of Mathematics is indexed by Mathematical Reviews, Zentralblatt MATH, PASCAL CNRS Index, Referativnyi Zhurnal, Current Mathematical Publications and Web of Knowledge (Science Citation Index).

The Pacific Journal of Mathematics (ISSN 0030-8730) at the University of California, c/o Department of Mathematics, 798 Evans Hall \#3840, Berkeley, CA 94720-3840, is published twelve times a year. Periodical rate postage paid at Berkeley, CA 94704, and additional mailing offices. POSTMASTER: send address changes to Pacific Journal of Mathematics, P.O. Box 4163, Berkeley, CA 94704-0163.

PJM peer review and production are managed by EditFLOW ${ }^{\circledR}$ from Mathematical Sciences Publishers.

\section{PUBLISHED BY}

\section{mathematical sciences publishers \\ nonprofit scientific publishing}

http://msp.org/

(C) 2016 Mathematical Sciences Publishers 


\section{PACIFIC JOURNAL OF MATHEMATICS}

Volume $280 \quad$ No. $1 \quad$ January 2016

Stable capillary hypersurfaces in a wedge

JAIGYOUNG CHOE and MIYUKI KoISO

The Chern-Simons invariants for the double of a compression body

DAVID L. DUNCAN

Compactness and the Palais-Smale property for critical Kirchhoff equations in

closed manifolds

EMMANUEL HeBEy

On the equivalence of the definitions of volume of representations

SUNGWOON KIM

Strongly positive representations of even GSpin groups

YEANSU KIM

An Orlik-Raymond type classification of simply connected 6-dimensional torus manifolds with vanishing odd-degree cohomology

\section{SHINTARÔ KUROKI}

Solutions with large number of peaks for the supercritical Hénon equation

Zhongyuan LiU and ShuangJie Peng

Effective divisors on the projective line having small diagonals and small heights and 141 their application to adelic dynamics

YÛSUKE OKUYAMA

Computing higher Frobenius-Schur indicators in fusion categories constructed from inclusions of finite groups

\section{Peter Schauenburg}

Chordal generators and the hydrodynamic normalization for the unit ball

SEBASTIAN SCHLEISSINGER

On a question of A. Balog

ILYA D. SHKREDOV

Uniqueness result on nonnegative solutions of a large class of differential inequalities 241 on Riemannian manifolds

YUHUA SUN

Correction to "Closed orbits of a charge in a weakly exact magnetic field"

WILL J. MERRY 